\title{
GERMINAÇÃO DE SEMENTES DE TOMATE SUBMETIDAS A DIFERENTES CONCENTRAÇÕES DE CLORETO DE SÓDIO E SUBSTRATOS
}

\author{
$\underline{\text { Rubiana Pereira Borges }}^{1}$ Lilian Thurler Nogueira ${ }^{1}$ Daiane Cecchin $^{2}$ Carlos Rodrigues \\ Pereira ${ }^{2}$ Cristina Moll Hüther ${ }^{3}$
${ }^{1}$ Mestranda do Programa de Pós-Graduação em Engenharia de Biossistemas da Universidade Federal Fluminense-UFF,(rubianapb@gmail.com) Niterói, Brasil Federal Fluminense-UFF, Niterói, Brasil Universidade Federal Fluminense-UFF, Niterói, Brasil \\ ${ }^{2}$ Docente no Departamento de Engenharia Agrícola e Meio Ambiente da Universidade \\ ${ }^{3}$ Pós-Doutoranda do Programa de Pós-Graduação em Engenharia de Biossistemas da
}

Recebido em: 22/09/2018 - Aprovado em: 23/11/2018 - Publicado em: 03/12/2018 DOI: 10.18677/EnciBio_2018B49

\begin{abstract}
A salinidade do solo é um fator limitante de produção, sendo proveniente desde a origem do solo, pluviosidade irregular e manejo inadequado da irrigação, podendo afetar desde a germinação, formação de plântulas, bem como plantas em estádio vegetativo e reprodutivo, no entanto, isto pode variar de espécie para espécie, bem como em diferentes variedades. Sendo assim, o objetivo deste trabalho foi avaliar a influência de diferentes concentrações de $\mathrm{NaCl}$ na germinação de sementes de tomate da variedade Santa $\mathrm{Cruz} \AA$ em diferentes tipos de substratos. Para isso, o experimento foi conduzido em casa de vegetação onde foram semeadas as sementes em dois substratos: solo arenoso e terra vegetal. As doses do sal, aplicadas no dia da semeadura, foram: 0; 8,75 (25\%); 17,5 (50\%); 26,25 (75\%); 35 $(100 \%) \mathrm{g} \mathrm{L}^{-1}$ de $\mathrm{NaCl}$, sendo que a dose de $100 \%$ equivalente a concentração de sal na água do mar. O solo arenoso apresentou maior IVG, índice de velocidade e germinação, e porcentagem de germinação, sendo que quanto maior a concentração de $\mathrm{NaCl}$, menores esses índices. $\mathrm{NaCl}$ foi um fator limitante na germinação de sementes de tomate variedade Santa Cruz® e existe uma interação entre as doses de $\mathrm{NaCl}$ e o tipo de substrato.
\end{abstract}

PALAVRAS-CHAVE: estresse salino, índice de velocidade de germinação (IVG), solo arenosos.

\section{GERMINATION OF TOMATO SEEDS SUBMITTED TO DIFFERENT CONCENTRATIONS OF SODIUM CHLORIDE AND SUBSTRATES}

\begin{abstract}
The salinity of the soil is a limiting factor of production, being from the origin of the soil, irregular rainfall and inadequate management of the irrigation, being able to affect from the germination, seedling formation, as well as plants in vegetative and reproductive stage, however, this can vary from species to species, as well as in different varieties. Therefore, the objective of this work was to evaluate the influence of different concentrations of $\mathrm{NaCl}$ on the germination of Santa Cruz ${ }^{\circledR}$ tomato seeds on different types of substrates. For this, experiment was conducted in a greenhouse where the seeds were sown in two substrates: sandy soil and vegetal soil. The doses ENCICLOPÉDIA BIOSFERA, Centro Científico Conhecer - Goiânia, v.15 n.28; p.571


of the salt, applied on the sowing day, were: 0; 8.75 (25\%); $17.5(50 \%) ; 26.25(75 \%)$; $35(100 \%) \mathrm{g} \mathrm{L}-1$ of $\mathrm{NaCl}$, the dose being $100 \%$ equivalent to the salt concentration in seawater. The sandy soil presented higher IVG and germination percentage, and the higher the $\mathrm{NaCl}$ concentration, the lower these indices. $\mathrm{NaCl}$ was a limiting factor in the germination of Santa Cruz ${ }^{\circledR}$ tomato seeds and there is an interaction between the $\mathrm{NaCl}$ doses and the substrate type.

KEYWORDS: germination rate index (GSI), saline stress, sandy soil

\section{INTRODUÇÃO}

O Brasil planta cerca de $66,5 \%$ de toda hortaliça mundial, sendo que o tomate está dentre as mais cultivadas. Os custos de produção da cultura são altos, destacando-se os custos com sementes que podem chegar a $10 \%$ do valor de produção (TREICHEL et al., 2016; CAMARGO FILHO ; CAMARGO, 2017).

Deste modo fatores genéticos, bióticos e abióticos são capazes de afetar a qualidade das sementes e esses efeitos são capazes de diminuir a germinação, crescimento e fotossíntese nas plantas, sendo que as sementes com maior qualidade, apresentam, na teoria, capacidade superior às outras de tolerar fatores desfavoráveis (SILVA et al., 2016; EBRAHIM; SALEEM, 2017).

$\mathrm{Na}$ obtenção de mudas de hortaliças o uso de substrato vegetal é comumente utilizado, podendo causar contaminação tanto por patógenos como conter uma elevada quantidade de sais que podem prejudicar a germinação de sementes (NOVAIS et al., 2007; LUDWIG et al., 2014). Contudo um dos principais estresses ambientais responsável por perdas de produtividade, o estresse por salinidade, vem sendo importante causador de danos fisiológicos nas plantas e 0 tomate considerado moderadamente sensível a este (ZUSHI; MATSUZOE, 2017).

A salinidade dos solos pode ser provocada por deficiência hídrica, distribuição desigual da precipitação, lençol freático raso, salinização da água do mar ou pelo material de origem que provocam o acúmulo de sais que prejudicam o crescimento vegetal (NOVAIS et al., 2007; ZHANG et al., 2018).

Os sais contidos na solução do solo diminuem o potencial osmótico da semente reduzindo a absorção de água e a concentração de íons no protoplasma, induzindo modificações metabólicas nas plantas, e influenciando negativamente na taxa de germinação (CONUS et al., 2009; PEDROTTI et al., 2015). Sendo assim o objetivo deste trabalho foi avaliar a interação entre diferentes concentrações de $\mathrm{NaCl}$ em dois substratos diferentes na germinação de sementes de tomate da variedade Santa Cruz®.

\section{MATERIAL E MÉTODOS}

O experimento foi realizado em casa de vegetação, localizada no Campus Gragoatá da Universidade Federal Fluminense, em Niterói, onde foram semeadas sementes de tomate da variedade Santa Cruz $\AA^{\circ}$, em bandejas de 200 células de polietileno, em dois substratos diferentes: solo arenoso e terra vegetal comercial Verde Vida ${ }^{\circledR}$. Em um delineamento inteiramente casualizado, com fatorial $(2 \times 5)$, sendo o fator A tipos de substratos e o fator $B$ as doses de sal.

Após a semeadura foi aplicada a solução salina em todas as células contendo as sementes de tomate com $2 \mathrm{~mL}$ de solução contendo as concentrações de $0 ; 8,75$ (25\%); 17,5 (50\%); 26,25 (75\%); 35 (100\%) g L ${ }^{-1}$ de NaCl, permaneceram por seis horas até a primeira irrigação com água normal. Sendo a dose de $100 \%$ equivalente a concentração do mar (SZIKSZAY, 1993). Cada unidade amostral foi formada por 
10 plantas e 10 repetições. A taxa de germinação foi aferida todos os dias, até o décimo quinto dia, quando todos os tratamentos já não apresentavam mais sementes germinando.

Determinou-se a porcentagem de germinação e o Índice de Velocidade de Germinação (IVG). A porcentagem de germinação foi calculada pela fórmula proposta nas Regras para Análise de Sementes (BRASIL, 2009) e o IVG de acordo com a fórmula proposta por Maguire (1962) adaptado de Moraes et al. (2012). Os dados foram submetidos à análise de variância com auxílio do programa SISVAR $\AA^{\circ}$ (FERREIRA, 2014), e as médias comparadas pelo teste de Tukey, a 5\% de probabilidade.

\section{RESULTADOS E DISCUSSÃO}

O substrato, solo arenoso apresentou maior IVG e porcentagem de germinação, sendo que quanto maior a concentração de $\mathrm{NaCl}$, menores esses índices. Sendo assim, diferiu estatisticamente do substrato terra vegetal (Tabela 1).

TABELA 1: Índice de velocidade de germinação em diferente substrato.

\begin{tabular}{cc}
\hline Tratamentos & IVG \\
\hline Solo arenoso & $7,272 \mathrm{a}^{*}$ \\
Terra Vegetal & $4,306 \mathrm{~b}^{*}$ \\
\hline
\end{tabular}

Médias seguidas da mesma letra na coluna não diferem entre si pelo teste de Tukey a $5 \%$.

O resultado pode ser explicado devido ao fato de que a terra vegetal comercial pode ter diferentes origens do material utilizado na sua produção, o que interfere também na granulometria, pois Shao et al. (2014), quando estudaram diferentes materiais para obtenção de terra vegetal, encontraram diferenças na germinação de trigo devido ao material de origem dos substratos, no entanto, Ludwig et al. (2014), afirmam que diferentes substratos, com diferentes $\mathrm{pH}$, interferem na produção de matéria seca em plantas de gérbera, ou seja, estes dois fatores podem ter influenciado na diferença entre os tipos de substratos neste experimento.

Outro fator que também contribuiu para o menor IVG foi a textura do solo, pois, de acordo com Novais et al. (2007), a textura influencia nos efeitos negativos do $\mathrm{NaCl}$ sobre as culturas, sendo que solos com textura arenosa retém menos sais.

Quanto ao índice de velocidade de germinação, a dose $0 \%$ (controle) obteve o maior IVG, porém as doses de $25 \%$ e $50 \%$ não diferiram do controle. Sendo assim, conforme o aumento da dose de $\mathrm{NaCl}$, o IVG diminuiu, e nas doses $75 \%$ e $100 \%$ ficaram estatisticamente iguais entre si. (Tabela 2).

TABELA 2: Índice de velocidade de germinação em relação as doses de $\mathrm{NaCl}$ nos dois substratos.

\begin{tabular}{c|ccccc}
\hline Tratamentos & $0 \%$ & $25 \%$ & $50 \%$ & $75 \%$ & $100 \%$ \\
\hline IVG & $10,41 \mathrm{a}^{\star}$ & $8,05 \mathrm{ab}$ & $4,69 \mathrm{ab}$ & $3,39 \mathrm{~b}$ & $2,41 \mathrm{~b}$ \\
\hline
\end{tabular}

*Médias seguidas da mesma letra na linha, não diferem entre si pelo teste de Tukey a $5 \%$.

Assim sendo, para essa variedade de tomate, as doses de $\mathrm{NaCl}$ com 25 e $50 \%$ não afetaram de forma significativa a germinação das sementes, sugerindo uma possível tolerância frente a estas doses, no entanto, analisando o controle com 
maior dose de $\mathrm{NaCl}$, obteve-se redução de $76,85 \%$. Essa diminuição na germinação também foi evidenciada por Shaygan et al. (2017) em plantas de Atriplex halimus, que é considerada uma espécie vegetal tolerante a salinidade, encontraram diferença do controle para a dose de $-1,5 \mathrm{Mpa}$ de $\mathrm{NaCl}$ de $92,86 \%$ na germinação. Este resultado também foi observado por Conus et al. (2009) em experimento com sementes de milho expostas a vários sais, em que a germinação não foi afetada, mas o $\mathrm{NaCl}$ foi o que mais interferiu negativamente no vigor e desenvolvimento das plântulas de milho. O cloreto de sódio foi responsável por diminuir o IVG e aumentar plântulas anormais em sementes de morango (HARTER et al., 2014).

Outra forma de analisar os dados é a porcentagem de germinação, ou seja, a quantidade de plântulas germinadas ao longo dos dias após semeadura e que de acordo com Brasil (2009), na dose de $0 \mathrm{~g} \mathrm{~L}^{-1}$ de $\mathrm{NaCl}$, é determinado o máximo do potencial de germinação neste lote de sementes. Nesse aspecto o solo arenoso apresentou maior porcentagem de germinação e em menor tempo, iniciando-se no quinto dia e para o substrato terra vegetal somente no sétimo dia (Figura 1).
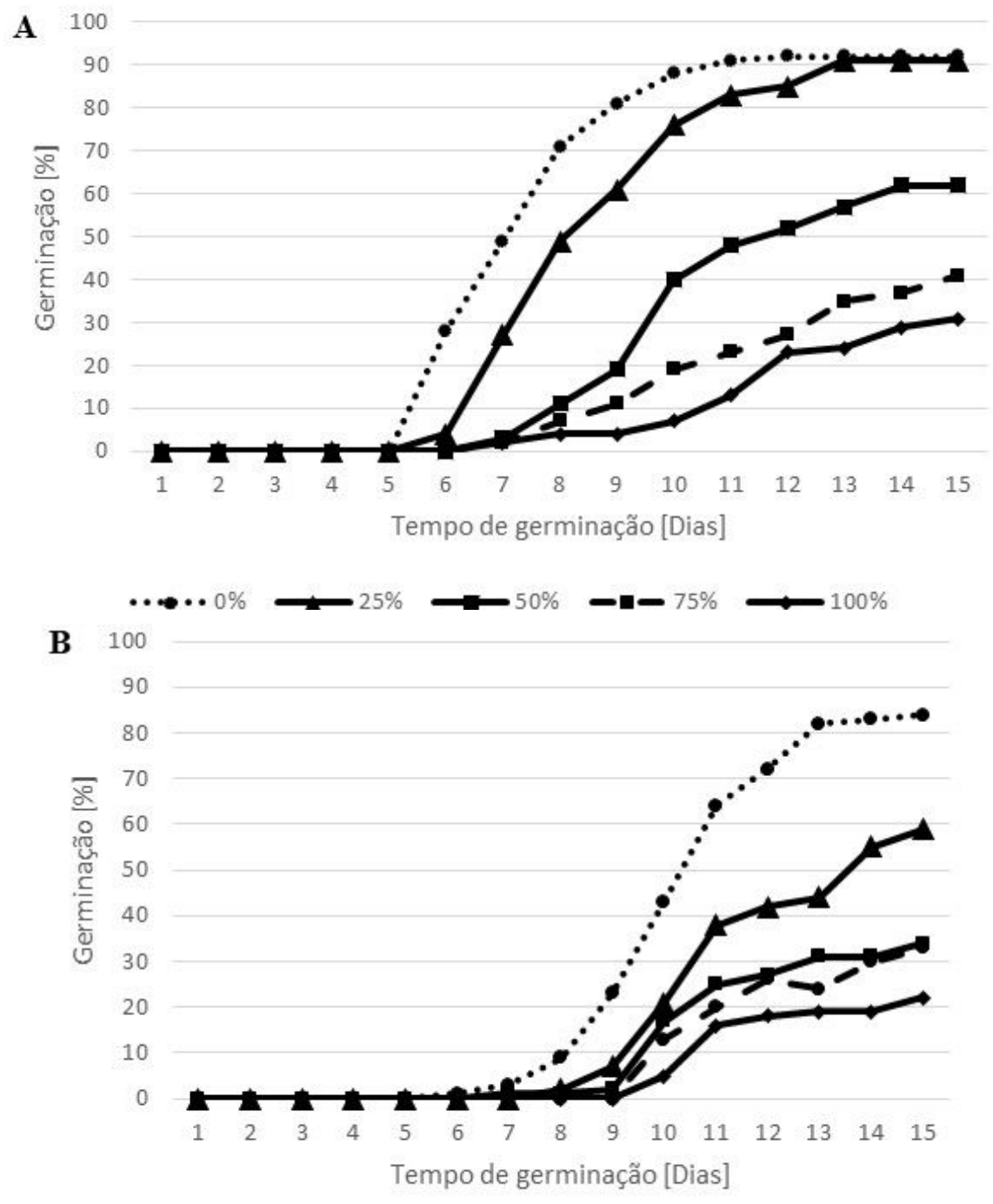

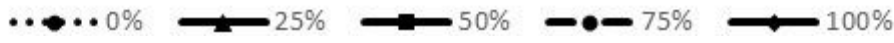

FIGURA 1: Porcentagem de germinação em diferentes sustratos em relação aos dias pós semeadura. Subtratos: (A) solo arenoso (B)terra vegetal. Doses de $\mathrm{NaCl}\left(\mathrm{g} \mathrm{L}^{-1}\right):(. . \bullet .) 0 \%.(\quad) 25 \%(\quad) 50 \%$ 
- ) $75 \%$ ( $100 \%$. Fonte: elaborado pelos autores.

Os resultados obtidos na porcentagem de germinação e IVG são semelhentes, pois a medida que houve o aumento das doses de $\mathrm{NaCl}$, concomitantemente a porcentagem de germinação diminuiu. $O$ solo arenoso apresentou maior porcentagem de germinação em todos os tratamentos e comparando os dois substratos no décimo dia após semeadura entre os controles, a diferença de germinação foi de $45 \%$. Contudo, na menor concentração de $\mathrm{NaCl}$, esta diferença apresentou-se $55 \%$ menor, o que justifica que o substrato terra vegetal é mais suscetível a todas as concentrações de $\mathrm{NaCl}$ no presente estudo.

Quando comparadas as duas maiores doses de $\mathrm{NaCl}$, a diferença entre os substratos foi de menos $10 \%$ o que comprovou que independente do substrato em altas concentrações o $\mathrm{NaCl}$ é prejudicial à germinação das sementes de tomate, atigindo o máximo de $19 \%$ na dose de $75 \%$ de $\mathrm{NaCl}$. Portanto, verificou-se que o substrato que menos interagiu com o $\mathrm{NaCl}$ foi o solo arenoso e que na medida que foram aumentadas as doses de $\mathrm{NaCl}$, o aumento foi proporcional na diminuição da germinação das sementes de tomate, indepedente do substrato.

\section{CONCLUSÃO}

$\mathrm{NaCl}$ é um fator limitante na germinação de sementes de tomate variedade Santa Cruz $\AA$, no entanto, dependendo do tipo de substrato, ocorre maior ou menor interação, o que afeta o índice de velocidade de germinação e porcentagem de germinação.

\section{AGRADECIMENTOS}

Agradecemos a CAPES e ao CNPq.

\section{REFERÊNCIAS}

BRASIL. Ministério da Agricultura, Pecuária e Abastecimento. Regras para análise de sementes. Ministério da Agricultura, Pecuária e Abastecimento. Secretaria de Defesa Agropecuária. Brasília: MAPA/ACS, 2009. 395p. Disponível em: http://www.agricultura.gov.br/assuntos/insumos-agropecuarios/arquivos-publicacoesinsumos/2946_regras_analise_sementes.pdf

CAMARGO FILHO, W.P.; CAMARGO, F.P. A quick review of the production and commercialization of the main vegetables in Brazil and the world from 1970 to 2015. Horticultura Brasileira, Vitória da Conquista, v.35, n.2, p.160-166, 2017. Disponível em: <http://dx.doi.org/10.1590/S0102-053620170202>. Acessado: Out. 02, 2017. doi: 10.1590/S0102-053620170202.

CONUS, L. A.; CARDOSO, P. C.; VENTUROSO, L. R.; SCALON, S. P. Q. Germinação de sementes e vigor de plântulas de milho submetidas ao estresse salino induzido por diferentes sais. Revista Brasileira de Sementes, v.31, n.4 p.6774, 2009. Disponível em: <http://dx.doi.org/10.1590/S0101-31222009000400008>. Acessado: Set. 26, 2017. doi: 10.1590/S0101-31222009000400008.

EBRAHIM, M.K.H.; SALEEM, A.R. Alleviating salt stress in tomato inoculated with mycorrhizae: Photosynthetic performance and enzymatic antioxidants. Journal of Taibah University for Science, v.11, n.6, p.850-860, 2017. Disponível em: 
<https://doi.org/10.1016/j.jtusci.2017.02.002>. Acessado: Set. 26, 2017. doi: 10.1016/j.jtusci.2017.02.002.

FERREIRA, D.F.; Sisvar: a Guide for its Bootstrap procedures in multiple comparisons. Ciência e Agrotecnologia, 2014, vol.38, n.2, pp. 109-112. Disponível em: <http://dx.doi.org/10.1590/S1413-70542014000200001>.

HARTER, L. S. H.; HARTER, F. S.; DEUNER, C.; MENEGHELLO, G.E.; VILLELA, A. $F$. Salinidade e desempenho fisiológico de sementes e plântulas de morango. Horticultura Brasileira, v. 32, p. 80-85, 2014. Disponível em: <http://dx.doi.org/10.1590/S0102-05362014000100013>. Acessado: Jan. 26, 2018. doi: 10.1590/S0102-05362014000100013.

LUDWIG, F.; FERNANDES, D. M.; GUERRERO, A. C.; VILLAS BÔAS, R. L. Características dos substratos na absorção de nutrientes e na produção de gérbera de vaso. Horticultura Brasileira, Vitória da Conquista, v.32, n.2, p.184-189, 2014. Disponível em: <http://dx.doi.org/10.1590/S0102-05362014000200011>. Acessado: Nov. 21, 2017. doi: 10.1590/S0102-05362014000200011.

MAGUIRE, J. D. Speed germination-aid in selection and evaluation for seedling emergence and vigor. Crop Science, v. 2, p. 176-177, 1962.

MORAES, D. M.; BANDEIRA, J. de M.; MARINI, P.; LIMA, M. G. S.; MENDES, C. R. Práticas laboratoriais em Fisiologia Vegetal. Pelotas: Editora Cópias Santa Cruz Ltda, 2012. 1v.162p.

NOVAIS, R. F.; ALVAREZ, V. H.; BARROS, N. F.; FONTES, R. L. F.; CANTARUTTI, R. B.; NEVES, J. C. L. Fertilidade do Solo. Viçosa: Sociedade Brasileira de Ciências do Solo, 2007. 1017p.

PEDROTTI, A.; CHAGAS, R. M.; RAMOS, V. C.; PRATA, A. P. N.; LUCAS, A. A. T.; SANTOS, P. B. S. Causas e consequências do processo de salinização dos solos. Revista Eletrônica em Gestão, Educação e Tecnologia Ambiental, Santa Maria, v. 19, n. 2, p. 1308-1324, 2015. Disponível em: <http://www.academia.edu/27479766/Causas_e_consequ\%C3\%AAncias_do_proces so_de_saliniza\%C3\%A7\%C3\%A30_dos_solos_Causes_and_consequences_of_the process_of_soil_salinization>. Acessado: Jan. ${ }^{-}$19, 2018. ${ }^{-}$doi: 105902/2236117016544.

SHAO, L.; FU,Y.; FU, W.; YAN, M.; LI, L.; LIU, H. Effects of aqueous extract of soillike substrate made from three different materials on seed germination and seedling growth of rice. Acta Astronautica. v.6, p.83-88, 2014. Disponível em: <https://doi.org/10.1016/j.actaastro.2013.10.007>. Acessado: Jan. 19, 2018. doi: 10.1016/j.actaastro.2013.10.007.

SHAYGAN, M.; BAUMGARTL, T.; ARNOLD, S.; Germination of Atriplex halimus seeds under salinity and water stress. Ecological Engineering, v.102, 
p.636-640, 2017. Disponível em: <https://doi.org/10.1016/j.ecoleng.2017.02.050>. Acessado Jan. 19, 2018. doi: 10.1016/j.ecoleng.2017.02.050.

SILVA, R.C.; GRZYBOWSKI, C. R. S.; PANOBIANCO, M. Vigor de sementes de milho: influência no desenvolvimento de plântulas em condições de estresse salino. Revista Ciência Agronômica, Fortaleza, v. 47, n. 3, p. 491-499, 2016. Disponível em: $\quad<h t t p: / / w w w . c c a r e v i s t a . u f c . b r / s e e r / i n d e x . p h p / c c a r e v i s t a / a r t i c l e / v i e w / 4177>$. Acessado: Jan. 19, 2018. doi: 10.5935/1806-6690.20160059.

SZIKSZAY, M. Geoquímica das Águas. São Paulo: Instituto de Geociências-USP, 1993, n.5, 166p. (Boletim técnico, 5).

TREICHEL, M. Anuário brasileiro do tomate. Santa Cruz do Sul: Editora Gazeta Santa Cruz, 2016. 84p.

ZHANG, M.; DONG, B; QIAO, Y.; YANG, H.; WANG, Y.; LIU, M. Effects of sub-soil plastic film mulch on soil water and salt content and water utilization by winter wheat under different soil salinities. Field Crops Research v.225 p. 130-140 (2018) Disponível em: https://doi.org/10.1016/j.fcr.2018.06.010 Acessado: Ago. 02, 2018. doi.org/10.1016/j.fcr.2018.06.010.

ZUSHI, K.; MATSUZOE, N. Using of chlorophyll a fluorescence OJIP transients for sensing saltstress in the leaves and fruits of tomato. Scientia Horticulturae, v.219 p.216-221 (2017). Disponível em: https://doi.org/10.1016/j.scienta.2017.03.016 Acessado: Ago. 01, 2018. doi.org/10.1016/j.scienta.2017.03.016. 